\title{
Positive Ion Mobility in Normal and Superfluid ${ }^{3} \mathrm{He}^{*}$
}

\author{
J. Kokko, M. A. Paalanen, $\nmid$ W. Schoepe, $\$$ and Y. Takano \\ Low Temperature Laboratory, Helsinki University of Technology, Espoo, Finland
}

(Received March 21, 1978)

The mobility of positive ions has been measured in the normal and superfluid phases of ${ }^{3} \mathrm{He}$ at several pressures. Below $100 \mathrm{mK}$ the normal phase mobility increases logarithmically with decreasing temperature down to the superfluid transition temperature $\mathrm{T}_{c}$; it shows an anomalous jump near $100 \mathrm{mK}$. At low temperatures the drift velocity is nonlinear for electric fields exceeding $30 \mathrm{~V} / \mathrm{cm}$. In the superfluid the mobility, normalized to its value at $\mathrm{T}_{\mathrm{c}}$, is much less than for negative ions. We have also observed the anisotropic mobility in the A phase and the Landau critical velocity for pair-breaking in both superfluid phases.

In our previous measurements ${ }^{1.2}$ of negative ion mobility in liquid ${ }^{3} \mathrm{He}$, no trace of ionic recoil effects, which should reduce the scattering of quasiparticles at low temperatures, could be distinguished in the normal phase. Positive ions are expected to be lighter than negative ions, offering further insight into the effects of energy exchange between ${ }^{3} \mathrm{He}$ quasiparticles and the ion. For this reason we have extended the measurements to positive ions both in the normal and the superfluid phases. Indeed we have found that the positive ion mobility increases with decreasing temperature in the normal phase. In the superfluid phases we observed qualitatively similar behavior as in the case of negative ions. The enhancement of the mobility, normalized to the value at the superfluid transition temperature, however, was clearly less pronounced for positive ions. During the course of our work, Roach et al. ${ }^{3,4}$ reported similar experiments with negative and positive ions. We will compare their results with ours. Our normal phase data at low temperature, in particular, disagree with theirs and we will discuss the reasons for this discrepancy. Most recently Alexander et al. ${ }^{5}$ studied positive ions in the normal phase and observed novel features

*Work supported by the Academy of Finland.

$\dagger$ Present address: Bell Laboratories, Murray Hill, New Jersey.

$\doteqdot$ On leave of absence from Regensburg University, Regensburg, West Germany, supported by Deutsche Forschungsgemeinschaft. 
related to the creation of several ion species. Their mobility data cannot be directly compared with ours, because their electric fields were higher than ours by an order of magnitude.

The temperature independence of negative ion mobility was qualitatively understood in the framework of the theory by Josephson and Lekner. ${ }^{6}$ The positive ion results show an essential effect of temperature and should shed more light on the kinetics of ions in liquid ${ }^{3} \mathrm{He}$. The situation is somewhat similar in the case of the superfluid phases; the mobility calculation in the B phase, assuming elastic quasiparticle-ion collisions, by Baym et al., ${ }^{7}$ was in good agreement with experiment for negative ions, whereas our positive ion results seem clearly out of the range of validity of the theory.

The ${ }^{3} \mathrm{He}$ sample was cooled by nuclear demagnetization of copper. The temperature was determined by measuring the nuclear magnetic susceptibility and the spin-lattice relaxation time of platinum powder. Ions were produced by applying voltages between 800 and $2000 \mathrm{~V}$ to three tungsten field-ionization tips. Details of the refrigeration and thermometry, as well as the time-of-flight method of measuring the mobility, have been previously reported. ${ }^{2.8} \mathrm{An}$ advantage of the current apparatus is that the external magnetic field $(28 \mathrm{mT})$ could now be oriented to form any angle with the ion velocity. We were thus able to study the anisotropic mobility in the A phase. The drift space was $2.4 \mathrm{~mm}$ long. In order to estimate the absolute accuracy of our mobility data, we measured the negative ion mobility in the normal phase at 28 bar and obtained excellent agreement (within $4 \%$ ) with our previous value.

Figure 1a shows the temperature dependence of the positive ion mobility $\mu$ in the normal phase at 6 and 28 bar. Important features of the data are an anomalous jump in the mobility at about $100 \mathrm{mK}$ and the logarithmic increase toward decreasing temperatures, the increase being steeper at the higher pressure. We also found at the lowest temperature that the drift velocity $v$ was not proportional to the applied field $E$ at $E$ as small as $30 \mathrm{~V} / \mathrm{cm}$ (see Fig. 2). Care was taken therefore to determine the mobility from the ratio $v / E$ in the linear velocity regime. The data points at the lowest temperatures were obtained from the $v-E$ plots shown in Fig. 2. For each pressure we then went on using a safe field at higher temperatures, occasionally checking, by constructing the whole $v-E$ curve again, that increasing space charge effects had not warped the results. This led to higher allowed fields at higher temperatures. At 6 bar, for instance, we used $E=19 \mathrm{~V} / \mathrm{cm}$ for temperatures below $4.2 \mathrm{mK}$; the largest field was $51 \mathrm{~V} / \mathrm{cm}$. Roughly $20 \%$ of the points shown in Fig. 1a were taken from the $v-E$ plots.

Regarding the anomalous jump in the mobility, Roach et.al. ${ }^{4}$ have recently demonstrated that the anomaly is caused by tiny traces of ${ }^{4} \mathrm{He}$ 

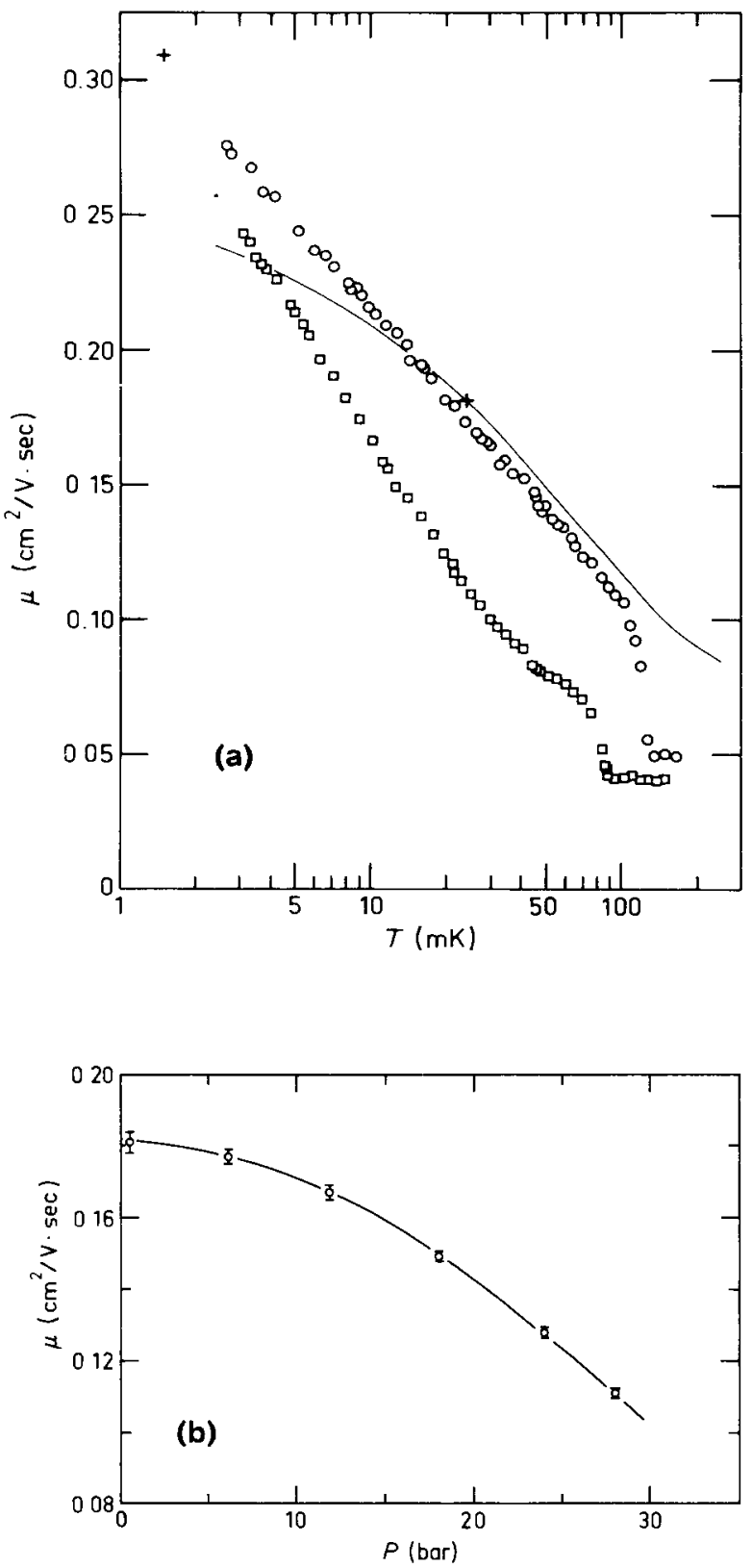

Fig. 1. Mobility of positive ions in the normal phase. (a) Temperature dependence of the mobility for $0.5(+), 6(0)$, and 28 bar $(\square)$. The solid line shows the smoothed data of Roach et al. ${ }^{4}$ (b) Pressure dependence at $24 \mathrm{mK}$. 


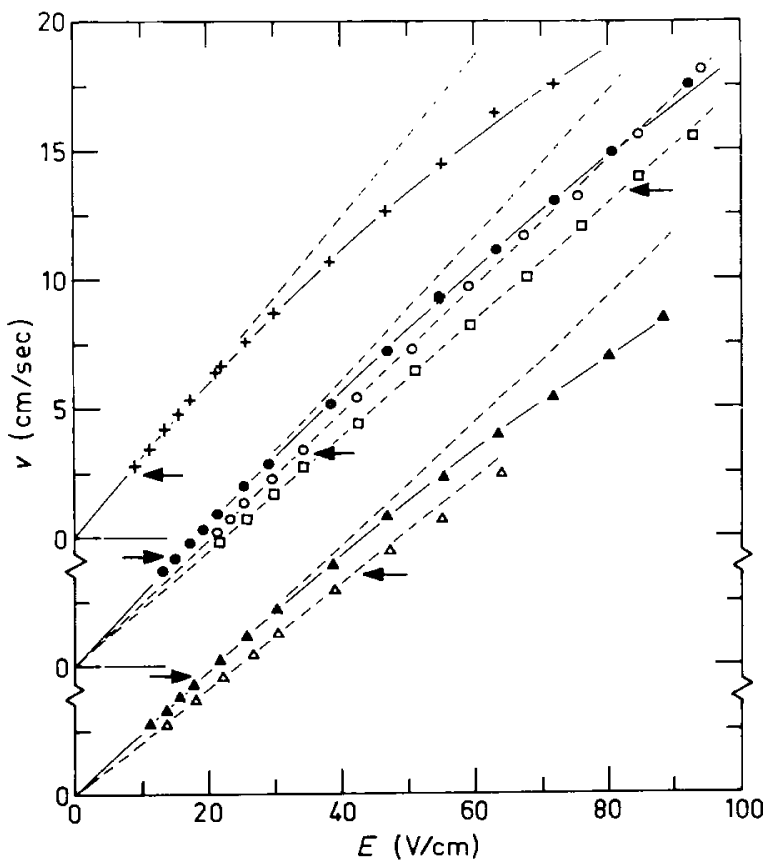

Fig. 2. Dependence of the drift velocity on the applied electric field in the normal phase. The inelastic threshold velocity $k_{\mathrm{B}} T / p_{\mathrm{F}}$ is indicated with arrows. Broken lines are extrapolations from the low-velocity region. $+, 1.5 \mathrm{mK}, 0.5$ bar. At 6 bar:, $2.66 \mathrm{mK} ; \bigcirc, 5.14 \mathrm{mK} ; \square, 8.42 \mathrm{mK}$. At 28 bar: $\Delta$, $3.11 \mathrm{mK} ; \triangle, 5.71 \mathrm{mK}$.

impurities in the sample, ${ }^{*}$ only at temperatures below the anomaly was the mobility unaffected by the impurity concentration. Therefore we will not dwell upon the temperature region above the anomaly.

The solid line in Fig. 1a shows the smoothed data of Roach et al. for their purest ${ }^{3} \mathrm{He}$ close to the vapor pressure. We have two data points at 0.5 bar marked by crosses in the figure. The data of Roach $e t$ al. agree with our point at $24 \mathrm{mK}$ but lie significantly lower than ours at $1.5 \mathrm{mK}$. Quite possibly they have underestimated the mobility by determining the ratio $v / E$ at too high an electric field. Their earlier observation ${ }^{10}$ that the

*The ${ }^{4} \mathrm{He}$ concentration in our sample was $30 \mathrm{ppm}$. The volume of the sample cell was $13 \mathrm{~cm}^{3}$ and the surface area of the copper sponge in the cell was $10-15 \mathrm{~m}^{2}$. By using a typical surface density of $10^{15}$ atoms $/ \mathrm{cm}^{2}$ for ${ }^{4} \mathrm{He}$, we estimate that the sponge surface absorbs as much as $400 \mathrm{ppm}$ of ${ }^{4} \mathrm{He}$ impurities in the sample until the monolayer coverage is completed. The mobility anomaly seems to be extremely sensitive to the minute concentration of ${ }^{4} \mathrm{He}$ left in the liquid due to thermal fluctuations. 
mobility was independent of electric field below $200 \mathrm{~V} / \mathrm{cm}$ is in disagreement with our results in Fig. 2. The mobilities obtained by Alexander et al. $^{5}$ from their fastest ion signals are qualitatively similar to ours, although their non-gating method introduces uncertainty in interpreting the signals.

The pressure dependence of the mobility at $24 \mathrm{mK}$ is shown in Fig. $1 \mathrm{~b}$. The mobility drops monotonically as the pressure increases and the change is more rapid at higher pressures. The same measurement for negative ions shows an opposite pressure dependence. ${ }^{2}$ The quite different behaviors of the two ion species are due to the remarkably different ion structures.* The negative ion is a "bubble" of vacuum containing an electron; the bubble radius shrinks with increasing pressure, which enhances the mobility. The negative ion radius obtained from our mobility measurements is in very good agreement with the values predicted by the bubble model. ${ }^{2}$ The positive ion, on the other hand, is a "snowball" of ${ }^{3} \mathrm{He}$ atoms solidified by the attractive electrostatic force of the positive charge. ${ }^{12}$ Since a higher pressure enhances the solidification, the ion radius increases at higher pressures; the decreasing mobility is due to this as well as to the fact that the Fermi momentum and the density of the quasiparticles increase toward higher pressures. The radius of positive ions is not quantitatively known.

One may try to estimate the positive ion radius with the aid of a result by Bowley, ${ }^{13}$ who fits the slope of the mobility as a function of $\ln T$ using the ion radius $R$ as a parameter. Taking the slope from the data below $60 \mathrm{mK}$ for 6 bar and below $20 \mathrm{mK}$ for 28 bar, we obtain $R=7.05$ and $6.18 \AA$. Contrary to the prediction of the snowball model, the radius is smaller for the higher pressure. Furthermore, taking all the parameters ${ }^{14}$ needed to fit the absolute value of our 6-bar data $\left(R=7.05 \AA, M / m_{3}=\right.$ $45 \pm 1$, the ratio of the ion mass to the ${ }^{3} \mathrm{He}$ atomic mass), we can use the theory to predict the mobility at 28 bar. The result at $3.1 \mathrm{mK}$ is $\mu=$ $0.178 \mathrm{~cm}^{2} / \mathrm{V} \mathrm{sec}, 27 \%$ lower than our experimental result. Increasing $R$ and $M$ would lower the prediction further. Obviously, then, the pressure dependence of the theory by Bowley is in disagreement with the snowball model. The larger slope of the higher pressure data can, however, be due to the temperature dependence of the ${ }^{3} \mathrm{He}$ melting pressure, which leads to temperature-dependent radii according to the snowball model. Neglecting the surface tension of the snowball, the model predicts $R(P) \propto$ $\left[n_{m} /\left(P_{m}-P\right)\right]^{1 / 4}$, where $P_{m}$ is the melting pressure, $P$ is the pressure of the liquid, and $n_{m}$ is the liquid density at the melting pressure. ${ }^{11,12}$ With the values from Halperin's data on the melting curve ${ }^{15}$ we estimate that the ion radius decreases by $13 \%$ when the liquid cools from 70 to $3 \mathrm{mK}$ at $28 \mathrm{bar}$; at 6 bar the decrease is only $2 \%$ in the same temperature range. A ${ }^{*}$ For a review on ion structures and mobilities see Fetter. ${ }^{11}$ 
decreasing ion radius not only reduces the number of colliding quasiparticles, but also makes the ion mass smaller, which enhances the ion's recoil. Both effects would tend to boost the growth of the mobility with decreasing temperature beyond what would happen with a constant radius. Thus, inferring the ion radius from the mobility data is not straightforward, particularly at high pressures.

Fetter and Kurkijärvi ${ }^{16}$ have pointed out that the drift velocity becomes nonlinear above the threshold velocity $k_{\mathrm{B}} T / p_{\mathrm{F}}$ for inelastic ionquasiparticle scattering, where $p_{\mathrm{F}}$ is the Fermi momentum. This critical velocity, as indicated by arrows in Fig. 2, seems to agree with the onset of the nonlinearity. We can express their prediction by the formula

$$
\mu E=v\left[1+a\left(p_{\mathrm{F}} v / k_{\mathrm{B}} T\right)^{2}+\cdots\right.
$$

where the coefficient $a$ depends on $\mu$. Since their explicit expression for the mobility $\mu$ is not self-consistent and does not agree with our data, we let $\mu$ and $a$ be fitting parameters and compare only the functional form with the experiment. Bowley has also used their theory for his model $^{13}$ and has numerically found the relation

$$
1 / v \simeq 1 / \mu^{\prime} E+1 / v_{\infty}
$$

for velocities exceeding $k_{\mathrm{B}} T / p_{\mathrm{F}}$, where the constants $\mu^{\prime}$ and $v_{\infty}$ depend on the temperature. The numerical values of the fitting parameters are listed in Table I, which should serve for checking the models. The fit with Eq. (2) was poor at low electric fields, where the proportionality between the drift velocity and the field holds. This is reflected in the fact that the coefficient $\mu^{\prime}$ in the table overestimates the mobility $\mu$. Note that in regimes where the drift velocity is not proportional to the field, Alexander et al. extracted the mobility by a fit equivalent to Eq. (2). We avoided such an uncertainty by determining the mobility strictly in the linear regime.

Figures $3 a$ and $3 b$ show the mobility in the superfluid phases divided by the value at $T_{c}$. All data were taken in the linear velocity region; we used no extrapolation from the nonlinear regime as was done by Roach $e t$ $a l^{3}$ The data at 28 bar were taken with the external magnetic field $\mathbf{B}$

TABLE I

\begin{tabular}{cccccccc}
\hline & & \multirow{2}{*}{$\begin{array}{c}\text { Low-velocity } \\
\text { value }\end{array}$} & \multicolumn{2}{c}{ Formula (1) } & & \multicolumn{2}{c}{ Formula (2) } \\
$P$, bar & $T, \mathrm{mK}$ & $\mu, \mathrm{cm}^{2} / \mathrm{V} \mathrm{sec}$ & $\mu, \mathrm{cm}^{2} / \mathrm{V} \mathrm{sec}$ & $a$ & & $\mu^{\prime}, \mathrm{cm}^{2} / \mathrm{V} \mathrm{sec}$ & $v_{\infty}, \mathrm{cm} / \mathrm{sec}$ \\
\hline 0.5 & 1.5 & 0.309 & 0.311 & $1.31 \times 10^{-2}$ & 0.327 & 74.3 \\
6 & 2.66 & 0.276 & 0.276 & $2.24 \times 10^{-2}$ & & 0.286 & 151 \\
28 & 3.11 & 0.243 & 0.244 & $5.24 \times 10^{-2}$ & & 0.253 & 110 \\
\hline
\end{tabular}


Positive Ion Mobility in Normal and Superfiuid ${ }^{3} \mathrm{He}$
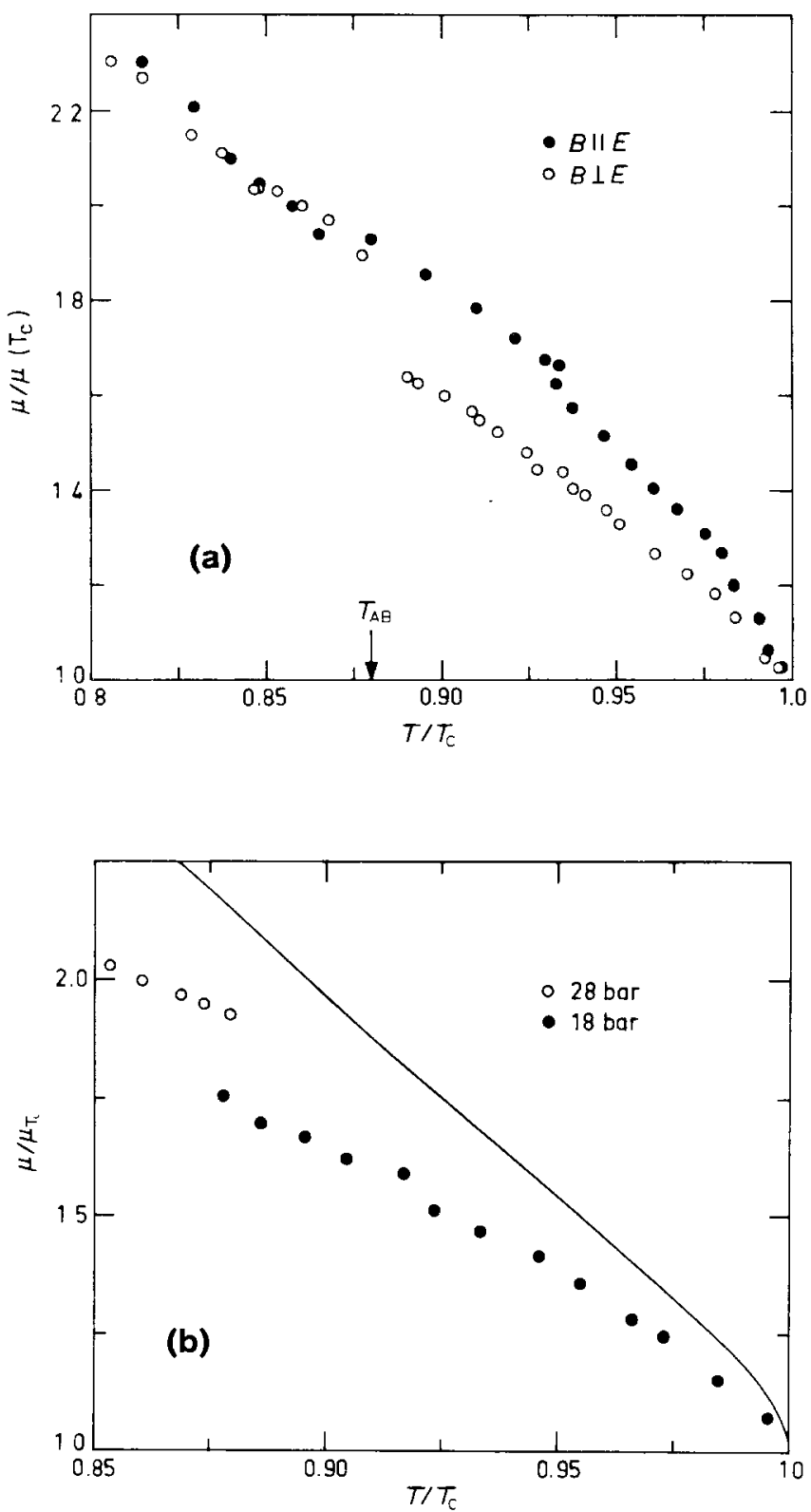

Fig. 3. (a) Mobility in the superfluid phases at 28 bar normalized to the value at $T_{c}$ for two orientations of the magnetic field. (b) Mobility in the $\mathbf{B}$ phase at 18 and 28 bar. The solid line is the calculation in Ref. 18. 
oriented both parallel and perpendicular to the electric field $\mathbf{E}$. We note the following features: Going down in temperature starting at $T_{c}$, the mobility starts rising rapidly. In addition, the mobility with $\mathbf{B} \| \mathbf{E}$ soon grows about $13 \%$ higher than with $\mathbf{B} \perp \mathbf{E}$. At the $A B$ transition the mobility with $\mathbf{B} \| \mathbf{E}$ goes smoothly over to the B-phase mobility within the experimental scatter. There appears to be a pressure dependence in the normalized mobility in the $B$ phase. In both phases the increase of the normalized mobility is considerably less than for negative ions; in the $\mathrm{B}$ phase, in particular, it is only one-half of the negative ion increase.

The anisotropy of the A-phase mobility agrees with the observation by Roach et al., ${ }^{3}$ except that the reduction of the mobility with $\mathbf{B} \perp \mathbf{E}$ is larger in our case. We believe that the discrepancy is due to a texture effect. Of the two configurations, only the one with $\mathbf{B} \| \mathbf{E}$ orients the anisotropic energy gap uniquely. The other leaves room for texture effects.

Normalizing the mobility to the value at $T_{c}$ is a reasonable procedure because the temperature dependence of the mobility in the normal phase is negligible compared to the superfluid phases. For instance at 28 bar, the normal phase mobility extrapolated into the superfluid would change only by $4 \%$ in the temperature range of our superfluid data. We measured the normal phase mobility at 18 bar only close to $T_{c}$. Effects such as an artificial pressure dependence in the normalized mobility could arise from the extrapolated growth being different from $4 \%$ at 18 bar. Such an uncertainty, however, _is not sufficient to account for the observed pressure dependence of the B-phase mobility. Note that no pressure dependence was observed for negative ions. ${ }^{2}$

Current theoretical treatments of ion mobility in the superfluid phases have mostly assumed an elastic ion-quasiparticle scattering model. For negative ions in the B phase the theory of Baym et al. ${ }^{7}$ agreed well with our previous experiments and demonstrated that the earlier calculations by Soda ${ }^{17}$ and by Bowley ${ }^{18}$ underestimated the mobility as a result of neglecting the superfluid effects on the scattering cross section. Our data for positive ions, on the other hand, lie lower than any of the theoretical predictions. The discrepancy is less for the theories assuming a constant scattering cross section; the result first derived by Bowley is shown in Fig. $3 \mathrm{~b}$ for comparison. This fact may suggest that the effect of the singularity in the density of quasiparticle states on the scattering cross section, which was envisaged by Baym et al., is overwhelmed by the energy exchange in the scattering process, leaving the number of thermally excited quasiparticles as the major difference between the normal and superfluid phases. Although a calculation for freely recoiling ions was recently done by Soda ${ }^{19}$ with a constant scattering cross section, the result showed no reduction from the elastic case. 


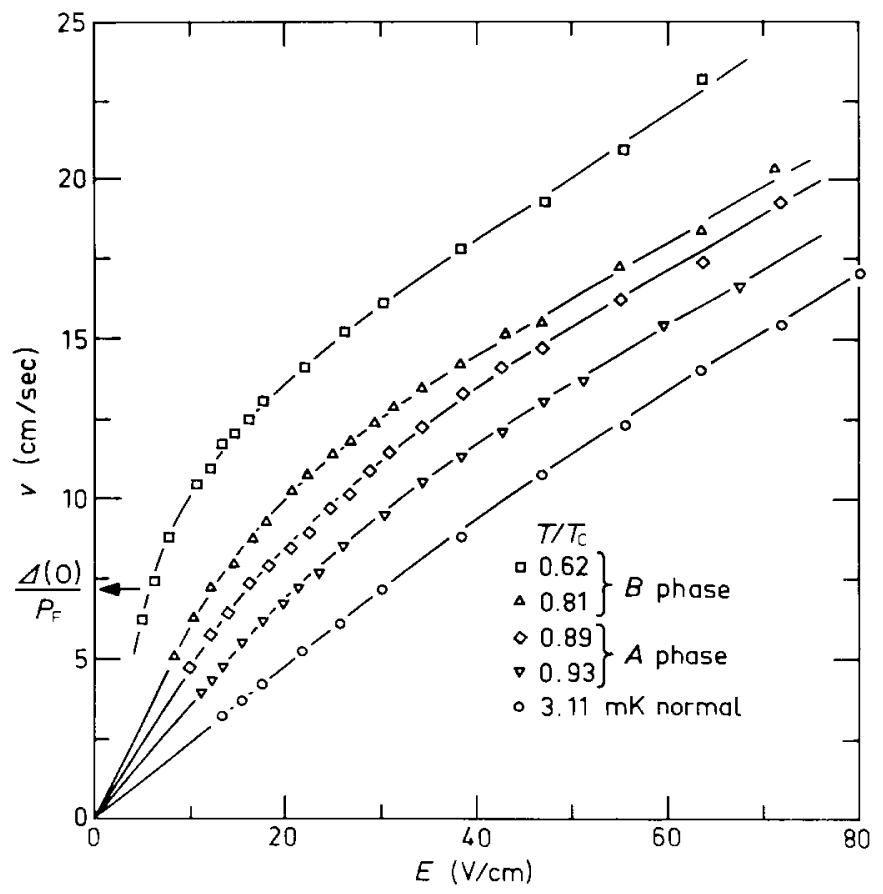

Fig. 4. Drift velocity as a function of the electric field in the superfluid at 28 bar. $\Delta(0) / p_{\mathrm{F}}$ is the Landau critical velocity for pairbreaking at zero temperature.

The nonlinearity of the drift velocity in the superfluid is illustrated in Fig. 4 , where data taken at 28 bar with $\mathbf{B} \| \mathbf{E}$ are shown. The features are similar to what one finds with negative ions: The onset of the nonlinearity is consistent with the Landau critical velocity for pair-breaking $\Delta / p_{F}$, where $\Delta$ is the superfluid energy gap. Above this threshold the slope becomes parallel to the normal phase data.

In summary, the mobility of positive ions in liquid ${ }^{3} \mathrm{He}$ at low temperatures shows remarkable dissimilarity to that of negative ions. The mobility is strongly temperature dependent in the normal phase and decreases with increasing.pressure. At low temperatures the drift velocity becomes nonlinear as a function of the applied electric field for unexpectedly small fields. In the superfluid the normalized mobility increases much less rapidly than for negative ions. A theoretical calculation qualitatively agrees with the temperature dependence of our normal phase mobility. Quantitative understanding of our superfluid results must await a theory that properly treats inelastic scattering. It is hoped that our data will stimulate further theoretical efforts in this direction. 


\section{ACKNOWLEDGMENTS}

We are grateful to Li Fu-zheng and Leila Rehn for valuable assistance during the measurements. We also appreciate stimulating discussions with Martti Salomaa, Henrik Smith, and especially Juhani Kurkijärvi, and helpful communications and suggestions from Roger Bowley, Mike Cross, and Doug Osheroff.

\section{REFERENCES}

1. A. I. Ahonen, J. Kokko, O. V. Lounasmaa, M. A. Paalanen, R. C. Richardson, W. Schoepe, and Y. Takano, Phys. Rev. Lett. 37, 511 (1976).

2. A. I. Ahonen, J. Kokko, M. A. Paalanen, R. C. Richardson, W. Schoepe, and Y. Takano, J. Low Temp. Phys. 30, 205 (1978).

3. P. D. Roach, J. B. Ketterson, and P. R. Roach, Phys. Rev. Lett. 39, 626 (1977).

4. P. D. Roach, J. B. Ketterson, and P. R. Roach, Phys. Lett. 63A, 273 (1977).

5. P. A. Alexander, C. N. Barber, P. V. E. McClintock, and G. R. Pickett, Phys. Rev. Lett. 39, 1544 (1977).

6. B. D. Josephson and J. Lekner, Phys. Rev. Lett. 23, 111 (1969).

7. G. Baym, C. J. Pethick, and M. Salomaa, Phys. Rev. Lett. 38, 845 (1977).

8. A. I. Ahonen, P. M. Berglund, M. T. Haikala, M. Krusius, O. V. Lounasmaa. and M. A. Paalanen, Cryogenics 16, 531 (1975).

9. J. G. Daunt, S. G. Hedge, and E. Lerner, in Monolayer and Submonolayer Helium Films, J. G. Daunt, and E. Lerner, eds. (Plenum Press, New York, 1973), p. 19.

10. P. D. Roach, J. B. Ketterson, and P. R. Roach, in Quantum Fluids and Solids, S. B. Trickey, E. D. Adams, and J. W. Dufty, eds. (Plenum Press, New York, 1977), p. 259.

11. A. L. Fetter, in The Physics of Liquid and Solid Helium, K. H. Bennemann and J. B. Ketterson, eds. (Wiley, New York, 1976), pp. 242-305.

12. K. R. Atkins, Phys. Rev. 116, 1339 (1959).

13. R. M. Bowley, J. Phys. C, to be published; and preprint.

14. R. M. Bowley, private communication.

15. W. P. Halperin, thesis, Cornell University 1975.

16. A. L. Fetter and J. Kurkijärvi, Phys. Rev. B 15, 4272 (1977).

17. T. Soda, Prog. Theor. Phys. 53, 903 (1975).

18. R. M. Bowley, J. Phys. C 2, L151 (1976).

19. T. Soda, Prog. Theor. Phys. 58, 1096 (1977). 